\title{
Evolution of syphilitic chancres with virulent Treponema pallidum in the rabbit
}

\author{
N. N. IZZAT, J. M. KNOX, J. A. WERTH, AND W. G. DACRES \\ Department of Dermatology and Syphilology, Baylor College of Medicine, Houston, Texas
}

In recent years there has been renewed interest in the immunology of syphilis and the possibility of developing a vaccine against this disease. The rabbit is the animal most frequently used for preliminary evaluation of vaccines. For this reason, we felt that a critical re-appraisal of the fate of Treponema pallidum when inoculated into the skin of normal rabbits was necessary. Early attempts to determine the lowest number of organisms necessary to produce infection gave varying results (Neisser, 1911; Chesney and Kemp, 1925; Wakerlin, 1926; Magnuson, Eagle, and Fleischman, 1948).

The last group found that intracutaneous inoculation of 2 to 200,000 virulent organisms of the Nichols strain of $T$. pallidum produced darkfield positive lesions in rabbits. The incubation period varied greatly with the size of the inoculum. Using the same virulent strain, we attempted to determine the minimal infective dose of $T$. pallidum and to study, clinically and pathologically, the evolution of chancres resulting from injecting specified numbers of spirochaetes.

\section{Material and methods}

New Zealand male rabbits weighing 5 to $6 \mathrm{lb}$. were used throughout the investigation. All were housed in individual cages in a specially designed room that kept the environmental temperature at precisely $70^{\circ} \mathrm{F}$. All animals had non-reactive serological tests for syphilis and had no evidence of infection with Treponema cuniculi. The virulent strain of $T$. pallidum used for inoculation was originally obtained from Dr. G. R. Cannefax of the National Communicable Disease Center, Atlanta, Georgia. This strain was maintained in the testes of normal rabbits and serially transferred every 11 days.

In a preliminary experiment four groups of four rabbits each were inoculated with varying numbers of spirochaetes. Chancres developed at all sites. Fig. 1 shows the relationship of the size of the inoculum to the average incubation period, which ranged from 9 days after injection of $25 \times 10^{3}$ organisms to 19 days after injection of fifty organisms.

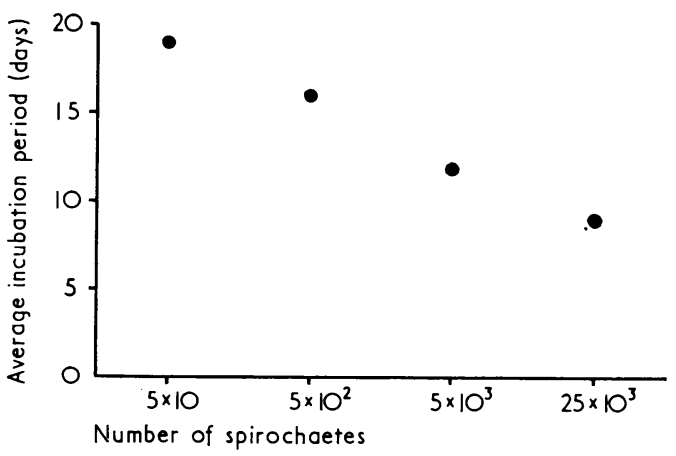

FIG. 1 Relationship of inoculum to incubation period in sixteen rabbits

In a larger series, 44 rabbits were inoculated with varying doses of spirochaetes ranging from nil to $10^{6}$ organisms. Each dose was injected in four sites on the backs of four different rabbits. Control rabbits received the suspension vehicle solution only.

PREPARATION OF TREPONEMAL SUSPENSION

Rabbit testes were removed 15 days after inoculation with $T$. pallidum (Nichols). The testes were finely minced, ground in a mortar, and mixed with $1 \mathrm{ml}$. physiological saline. The resulting emulsion was centifuged at $271 \mathrm{G}$. for 1 minute. The number of treponemes in the supernatant was determined by a modification of the method used by Magnuson and others (1948).

\section{CHALLENGE INOCULATION}

Unless otherwise stated, serial 10-fold dilutions of the calibrated supernatant were made in a $1: 1(\mathrm{v} / \mathrm{v})$ mixture of rabbit serum and non-pyrogenic saline to obtain final concentrations of from $10^{6}$ to 1 treponeme(s) per $0.1 \mathrm{ml}$. Graduated doses of organisms in $0.1 \mathrm{ml}$. volumes were inoculated intradermally into four sites on the back of each rabbit. These procedures were performed rapidly so that removal of rabbit testes, mincing, counting the treponemes, preparing the suspension, and inoculating the rabbits were completed within 20 minutes. Samples from $10^{6}$ suspension were injected into the testes of control rabbits to ensure that the strain remained infectious. 
After inoculation the sites on the back of each rabbit were examined daily for $\mathbf{4 0}$ days. Darkfield examinations were performed on every lesion that developed. Representative lesions which resulted from the introduction of ten to $10^{6}$ organisms per site were biopsied at 35 days after inoculation and the specimens were studied histologically using the haematoxylin and eosin and WarthinStarry staining techniques.

\section{Results}

The Table shows the incidence of darkfield positive lesions developing at sites injected with the various doses. All of the sites inoculated with ten to $10^{6}$ organisms were successfully infected. 63 per cent. of the sites inoculated with a suspension calibrated to contain five organisms were infected, but only 13 per cent. of the sites inoculated with 1 organism. The relationship between the size of the inoculum and the incubation period is shown in the Table and in Fig. 2. The mean incubation period with an inoculum of $10^{6}$ organisms was approximately 5 days. With each 10-fold decrease in the size of the inoculum there was a progressive lengthening of the incubation period. None of the control sites became infected. All animals inoculated intratesticularly with the $10^{6}$ doses of spirochaetes were found to be infected when darkfield examination was performed on the testicular tissue.

Because chancres developed at different times depending upon the size of the inoculum, the evolution of the chancres could be compared clinically and histopathologically on the same post-inoculation day. At 35 days the sites injected with ten organisms showed a small (12 mm.) circumscribed erythematous nodule (Fig. 3, opposite), whereas lesions developing after the injection of 25 organisms were larger and firmer and had a white halo about them (Fig. 4, opposite). Lesions which followed the inoculation of $10^{2}$ (100) organisms showed central necrosis with external crusting, and the skin around the base of these lesions was bluish-red in colour (Fig. 5). Inoculation of $10^{8}$ organisms resulted in much larger ulcerations at 35 days (Fig. 6). All the chancres subsequently healed

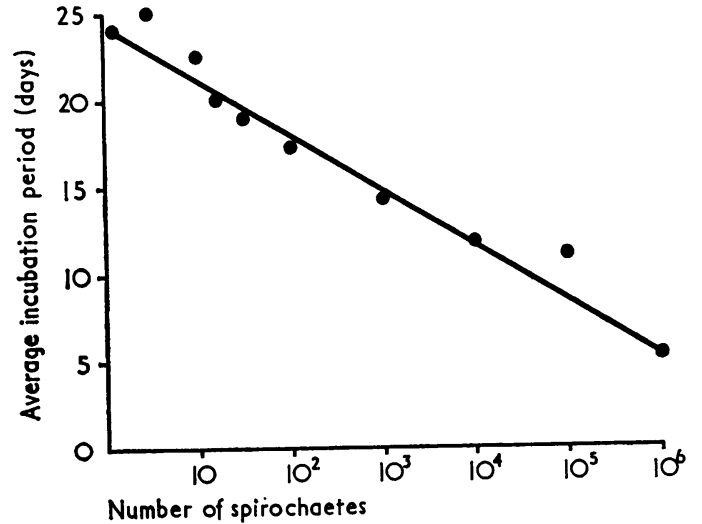

FIG. 2 Relationship of inoculum to incubation period in 44 rabbits

with re-growth of hair. The end-result was skin which appeared clinically normal.

Skin lesions from animals challenged with $10^{6}$ organisms were compared histopathologically with those challenged with only ten organisms at a constant time ( 35 days after inoculation). After 10 spirochaetes were injected the skin showed minimal epidermal crusting, sub-epidermal bulla formation, diffuse and marked oedema throughout the dermis, partial destruction of hair follicles, and a diffuse infiltrate primarily around the blood vessels. The infiltrate was composed of plasma cells, lymphocytes, histiocytes, and an occasional neutrophil (Fig. 7). With the Warthin-Starry stain, spirochaetes were seen mainly in the oedematous area beneath the epidermis, and to a lesser extent, in the epidermis, blood vessels, and deeper parts of the dermal connective tissue (Fig 8). When $10^{6}$ organisms were injected, the skin showed ulceration with complete to partial central necrosis. A small number of thickened and occluded blood vessels were present at the margin of the necrotic area. There was an infiltrate peripheral to and extending into the necrotic area (Fig. 9), and this was again composed of plasma cells, lympho-

TABLE Effect of the number of Treponema pallidum injected intracutaneously on the clinical appearance of lesions and on the incubation period

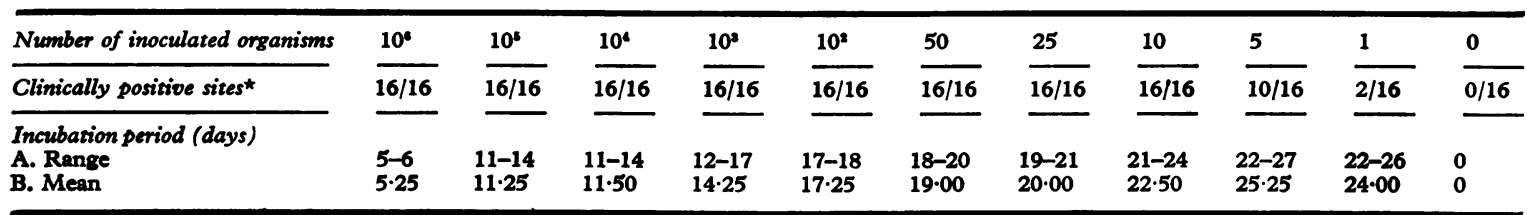

*Lesions are $9 \mathrm{~mm}$. or more in diameter and darkfield positive 


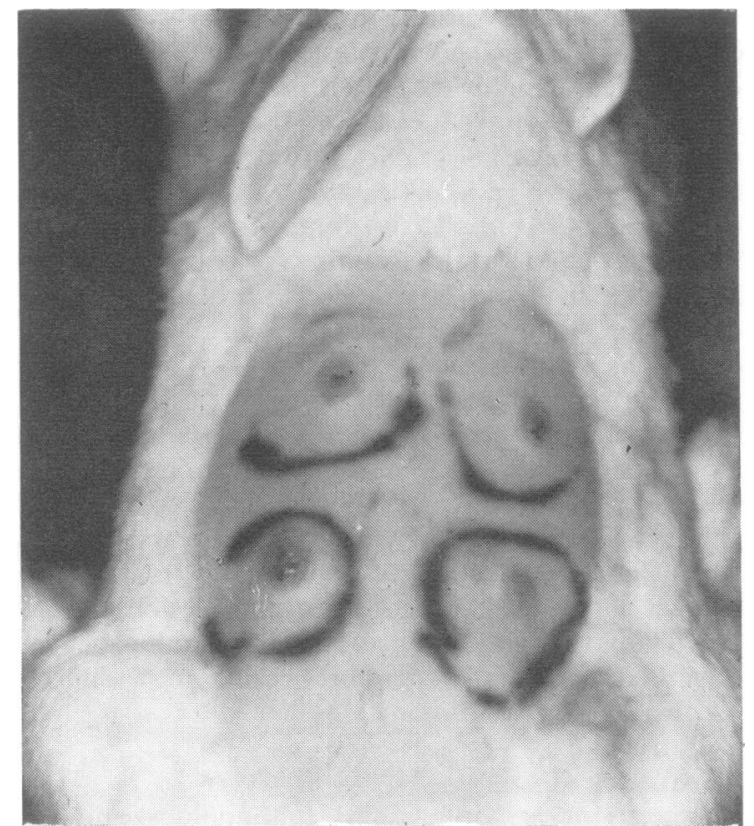

FIG. 3 Characteristic circumscribed syphilomata 35 days after intradermal inoculation with ten $\mathrm{T}$. pallidum (Nichols) organisms

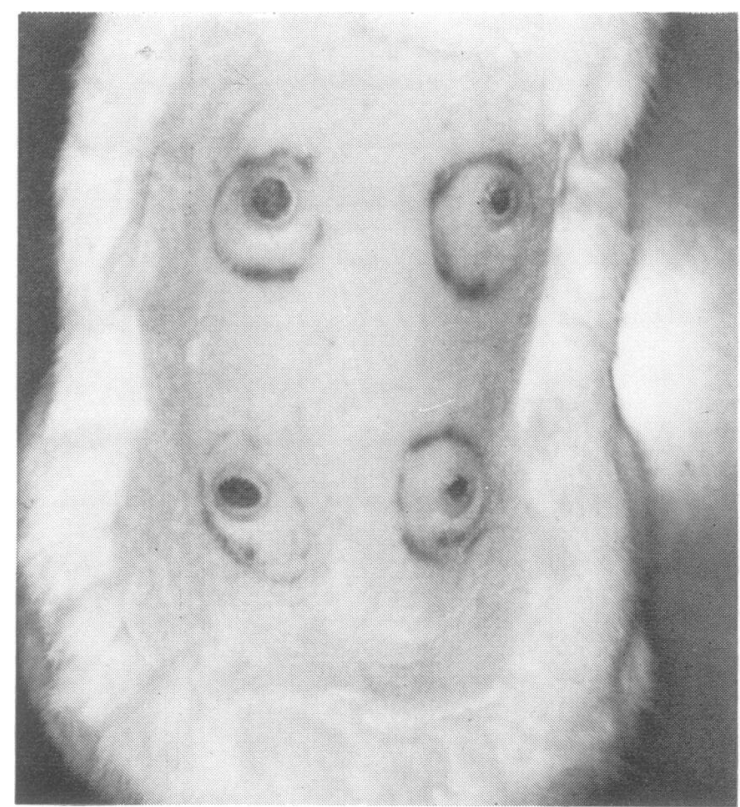

FIG. 4 Characteristic chancres with surrounding white halo after intradermal inoculation with $25 \mathrm{~T}$. pallidum (Nichols) organisms (35 days incubation)

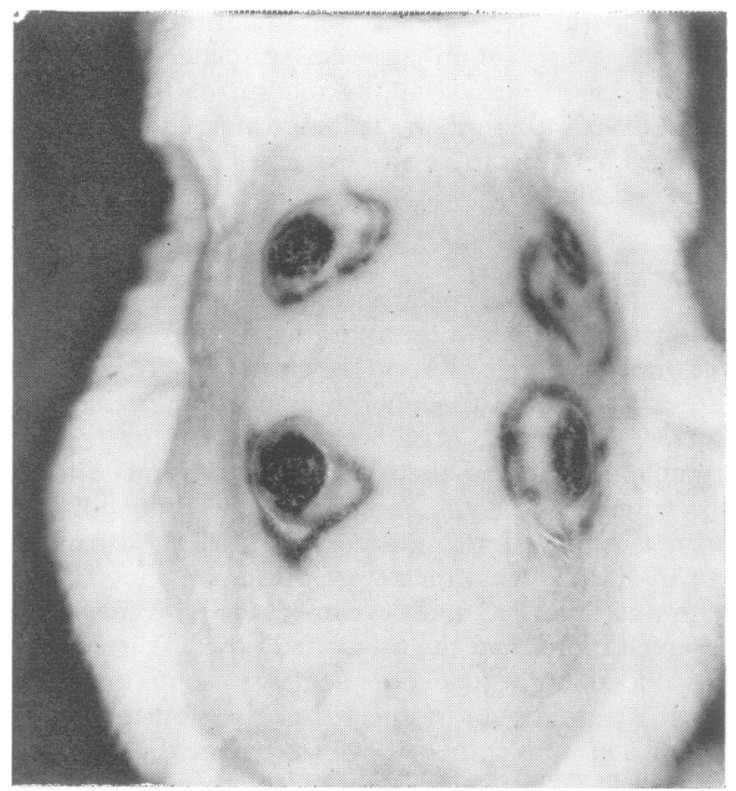

FIG. 5 Characteristic necrotic chancres of rabbit inoculated intradermally with $100 \mathrm{~T}$. pallidum (Nichols) organisms (35 days incubation)

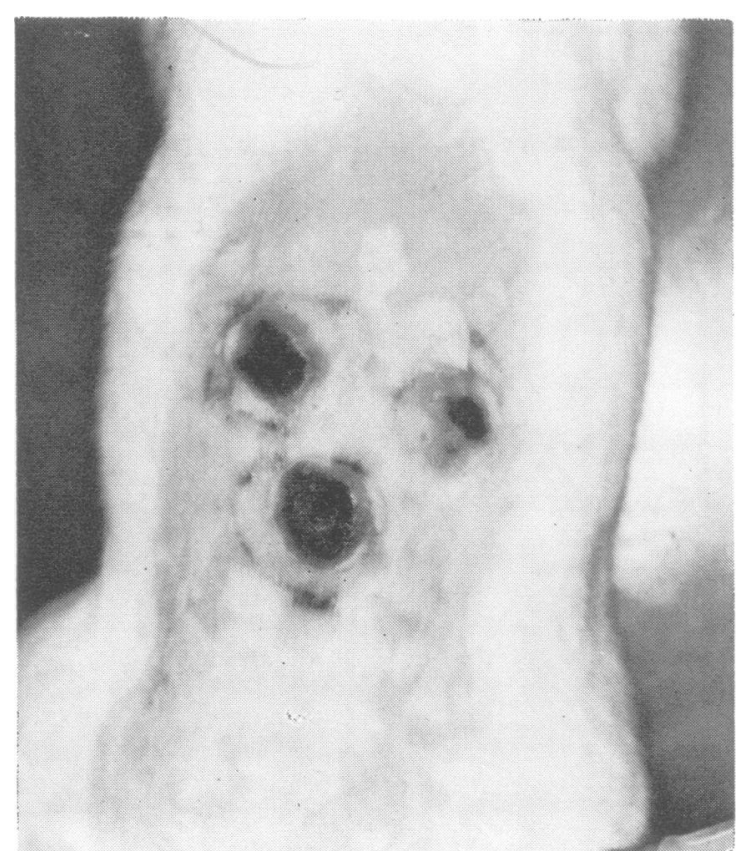

FIG. 6 Necrotic chancres of rabbit inoculated intradermally with 1,000,000 T. pallidum (Nichols) organisms per site (35 days incubation) 
cytes, histiocytes, and a few neutrophils. The Warthin-Starry stain showed spirochaetes in and around blood vessels at the margin of the necrotic area. With the other challenge dosage schedules, changes between these two extremes of the spectrum were revealed.

\section{Discussion}

After the intradermal inoculation of $T$. pallidum, incubation periods were found to vary inversely with the inoculum size. Also, larger inocula resulted in larger lesions. Although the incubation period was longer, small doses of spirochaetes $(10,5$, or 1$)$ were capable of inducing chancres. In suspensions diluted to contain five or one organism(s) the probability of error is high. It is quite possible that the two sites out of the sixteen which were presumably inoculated with one organism and developed chancres may have received more than the calculated dose. It is equally possible that the one organism was sometimes lost when we attempted to inject the other fourteen sites. Magnuson and others (1948), using the Nichols strain of $T$. pallidum, showed that as few as two organisms were sufficient to produce an infection when inoculated either intratesticularly or intracutaneously. Our data support their findings and reemphasize the highly infectious nature of $T$.pallidum.

Spirochaetes introduced into the skin multiply in a manner analogous to those observed in testicular tissue by Brown and Pearce (1920). As healing begins the number of spirochaetes diminishes. It should be noted that at 35 days the number of spirochaetes per highpower field was less in sites inoculated with $10^{6}$ organisms than in chancres resulting from ten organisms. This was unexpected in view of the larger size and more inflammatory character of the lesions.

The fact that large numbers of organisms in an inoculation result in a shorter incubation period in rabbit syphilis may explain the appearance of chancres at varying times after sexual contact in humans. A larger inoculation dose would probably result from contact with a well-developed primary lesion or with condylomata lata. Theoretically, an exposure of this type should therefore result in a shorter incubation period in man.

\section{Summary}

Renewed interest in syphilis immunology and the

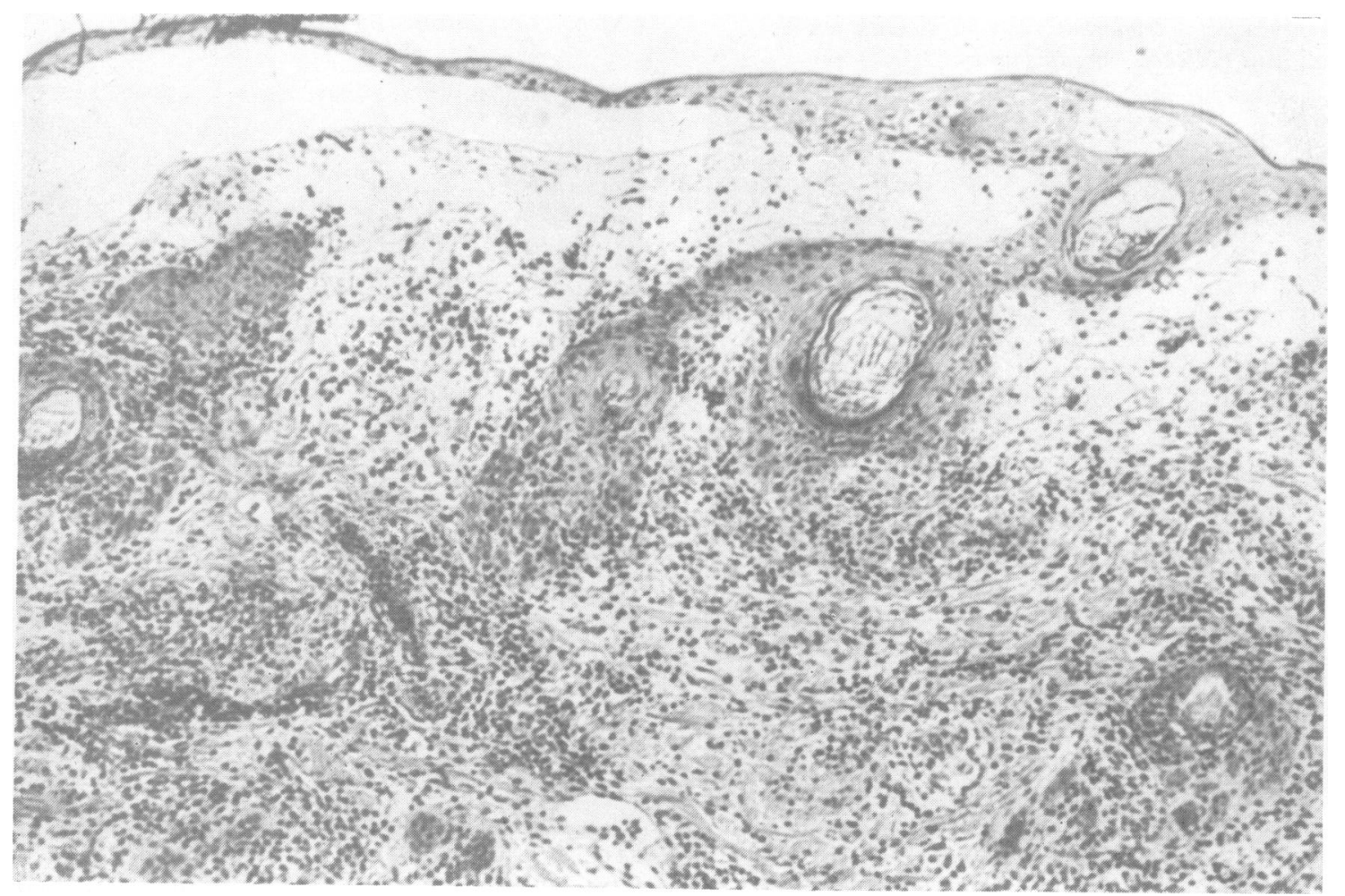

FIG. 7 Intradermal syphiloma in rabbit challenged with ten organisms. Haematoxylin and eosin. $\times 800$ 


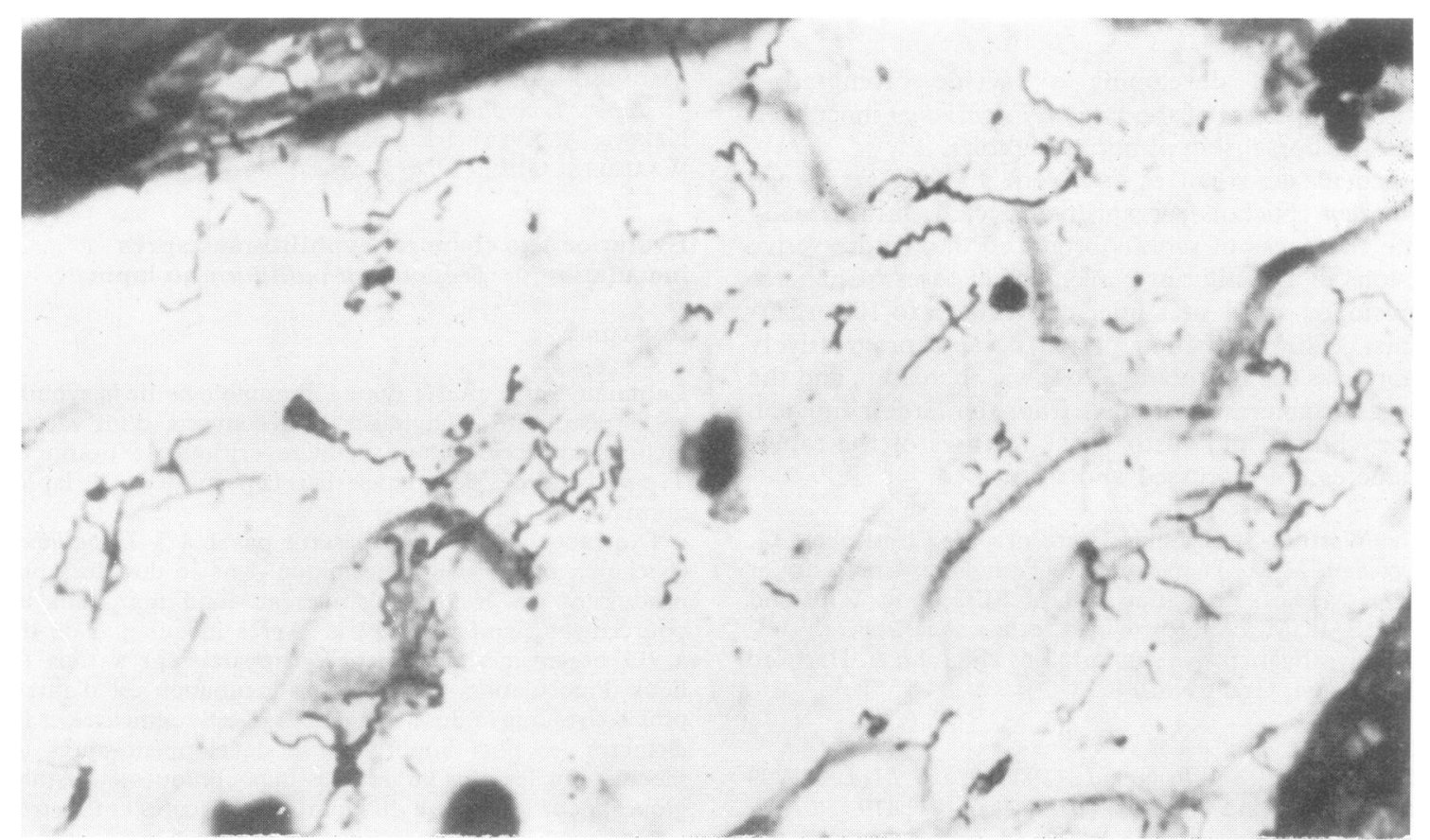

FIG. 8 Spirochaetes in chancres of rabbit challenged with ten organisms per site. Warthin-Starry stain. $\times 800$

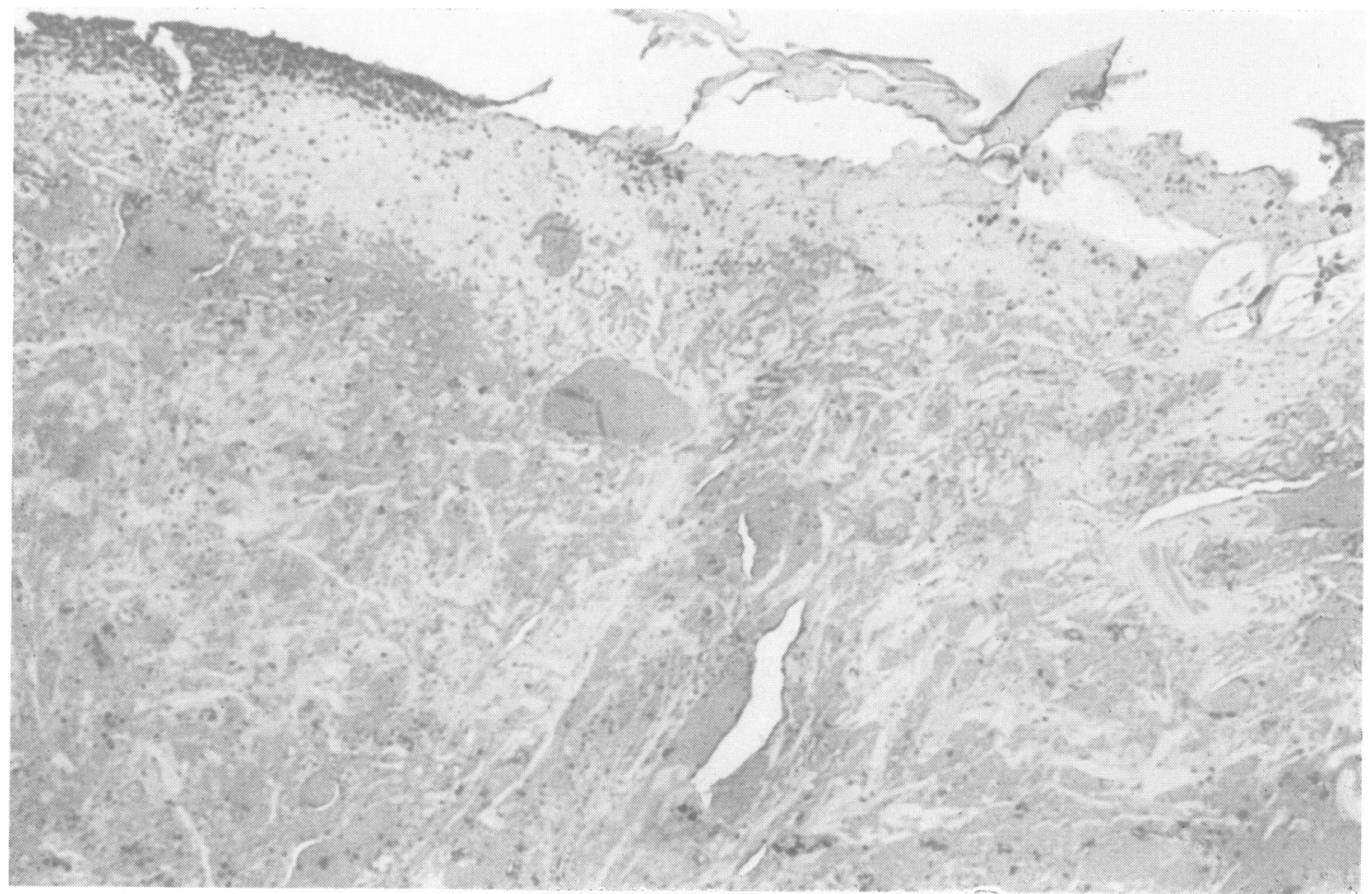

FIG 9 Necrotic chancre in rabbit inoculated with $10^{6}$ organisms. Haematoxylin and eosin. $\times 800$ 
possibility of developing a vaccine prompted a critical appraisal of the fate of $T$. pallidum inoculated into the dorsal skin of normal rabbits.

Inocula as small as from one to five Treponema pallidum (Nichols) organisms injected intradermally into the backs of rabbits produced darkfield positive lesions in a significant percentage of cases. Chancres developed at all sites injected with ten to $10^{6}$ organisms. The incubation period became progressively shorter as the inoculation dose was increased, and the largest chancres developed from the largest inocula. The clinical and histological features of the rabbit chancres are discussed and illustrated.

The Warthin-Starry slides were prepared by Robert G. Freeman, M.D., Department of Pathology, University of Texas Medical Branch at Dallas. Miss C. R. Wills and Miss S. E. McCotter provided technical assistance. The investigation was supported by the John A. Hartford Foundation, Grant No. 2465.

\section{References}

Brown, W. H., and Pearce, L. (1920) J. exp. Med., 31, 709

Chesney, A., and KeMP, J (1925) Ibid., 41479
Magnuson, H. J., Eagle, H., and Fleischman, R. (1948) Amer. F. Syph., 32, 1

NeIsSER, A. (1911) Arb. kaiserlich. Gesundh., 37, 56

WAKERLIN, G. E. (1926) f. infect. Dis., 38, 323

Evolution des chancres syphilitiques après inoculation de Treponema pallidum au lapin

SOMMAIRE

Le renouveau d'intérêt pour l'immunologie de la syphilis et la possibilité d'envisager la découverte d'un vaccin, incite à apprécier d'une manière critique le destin de $T$. pallidum après inoculation dans la peau du dos de lapins normaux.

Des inoculations, ne dépassant pas 1 à $5 T$. pallidum (Nichols), par voie intradermique dans le dos du lapin, produisent des lésions positives au fond noir dans un pourcentage significatif de cas. Après inoculation de dix à $10^{6}$ organismes, les chancres apparaissent à tous les lieux d'inoculation. La période d'incubation est d'autant plus courte que la dose d'inoculation est augmentée et les chancres les plus importants se développent après les inoculations les plus larges. Les faits cliniques et histologiques, pour le chancre du lapin, sont discutés et illustrés. 